\title{
INITIAL INVESTIGATION OF ENERGETIC PARTICLE PHENOMENA IN THE DISTANT MAGNETOTAIL FROM GEOTAIL/EPIC
}

\author{
A. T. Y. Lui, ${ }^{1}$ D. J. Williams, ${ }^{1}$ R. W. McEntire, ${ }^{1}$ C. Jacquey, ${ }^{1}$ \\ V. Angelopoulos, ${ }^{1}$ E. C. Roelof, ${ }^{1}$ S. M. Krimigis, ${ }^{1}$ C.-I. Meng, 1 \\ S. P. Christon, ${ }^{2}$ F. M. Ipavich, ${ }^{2}$ G. Gloeckler, ${ }^{2}$ T. P. Armstrong, 3 \\ L. J. Lanzerotti, ${ }^{4}$ E. T. Sarris, ${ }^{5}$ S. Kokubun, ${ }^{6}$ L. A. Frank, ${ }^{7}$ \\ K. L. Ackerson, ${ }^{7}$ W. R. Paterson, ${ }^{7}$ T. Yamamoto, ${ }^{8}$ T. Mukai ${ }^{8}$ \\ and $K$. Tsuruda 8 \\ 1 The Johns Hopkins University Applied Physics Laboratory, Laurel, \\ MD 20723, U.S.A \\ 2 Depariment of Physics, University of Maryland, College Park, MD 20742, \\ U.S.A. \\ 3 Department of Physics, University of Kansas, Lawrence, KA 66044, U.S.A. \\ ${ }^{4}$ AT\&T Bell Laboratories, 600 Mountain Avenue. Murray Hill, NJ 07974. \\ U.S.A. \\ ${ }^{5}$ The University of Thrace, Xanthi, Greece \\ 6 Solar-Terrestrial Environment Laboratory, Nagoya University, \\ Toyokawa 442, Japan \\ 7 Department of Physics, University of lowa, Iowa City, IA 52242, U.S.A. \\ 8 The Institute of Space and Astronautical Science, Kanagawa 229, Japan
}

\begin{abstract}
An initial survey of distant tail energetic particle phenomena from Geotail shows that (1) energetic ions of both solar wind and ionospheric origin populate the distant tail, (2) the entry of energetic ions from the magnetosheath into the magnetotail can take place many tens of earth radii behind the Earth in a transient manner, (3) energetic molecular ions of ionospheric origin are present in the distant tail during very high geomagnetic activity, and (4) energetic ion flux enhancements and substorm intensifications are temporally correlated.
\end{abstract}

\section{INTRODUCTION}

Geotail was launched in July 24,1992 , to become the first satellite in orbit for the International Solar Terrestrial Physics (ISTP) program. After a period of initial checkouts for all the instruments on the satellite, the Energetic Particles and Ion Composition (EPIC) instrument began its full operation on September 29, 1992. EPIC has two detector components: the Ion Composition Sensor (ICS) and the Supra-Thermal Ion Composition Spectrometer (STICS), as described in detail by Williams et al. /1/. Both ICS and STICS provide flux, composition, spectral, and angular distribution for all major elemental species from protons through iron. ICS measures ions from $\sim 50 \mathrm{keV}$ to $3 \mathrm{MeV}$ along with electrons $>32 \mathrm{keV}$ and $>110 \mathrm{keV}$ while STICS covers 
ions from $30 \mathrm{keV}$ to $230 \mathrm{keV} / \mathrm{e}$ with charge state information. ICS samples the angular distribution of ions at two polar angles and that of electrons at the satellite spin plane. STICS covers nearly the entire polar angle range with six heads.

One of the goals of the Geotail mission is to elucidate the transfer of mass, momentum, and energy between different plasma domains in the Geospace system. In this paper, we review our initial effort in addressing this issue through an examination of energetic phenomena in the distant tail based primarily on the EPIC measurements.

\section{ENERGETIC ION POPULATION IN DISTANT TAIL BOUNDARY LAYER}

Geotail detected a series of energetic particle flux enhancements near the apogee of its $A 6$ orbit when the spacecraft was located at $(X, Y, Z) \approx(-81,-8,9) R_{\mathrm{E}}$ in GSM coordinates, as reported by Williams et al, /2/. The energetic particle, magnetic field, and plasma measurements during this period of 0300-0600 UT on 1992 October 27 are shown in Fig. 1. The panels, from top to bottom, show the spin-averaged fluxes of 58$77 \mathrm{keV}$ protons, 70-96 keV helium ions, 186-221 keV oxygen ions, polar and azimuthal angles of the magnetic field, magnetic field variability, the plasma density, and the $x$-component of the plasma bulk flow. The spacecraft location is shown in the $X Y$ - and $X Z$-plane projections at the bottom of the figure. There is a clear correlation between these measurements. For instance, in the 0315-0335 UT interval, the first maxi-

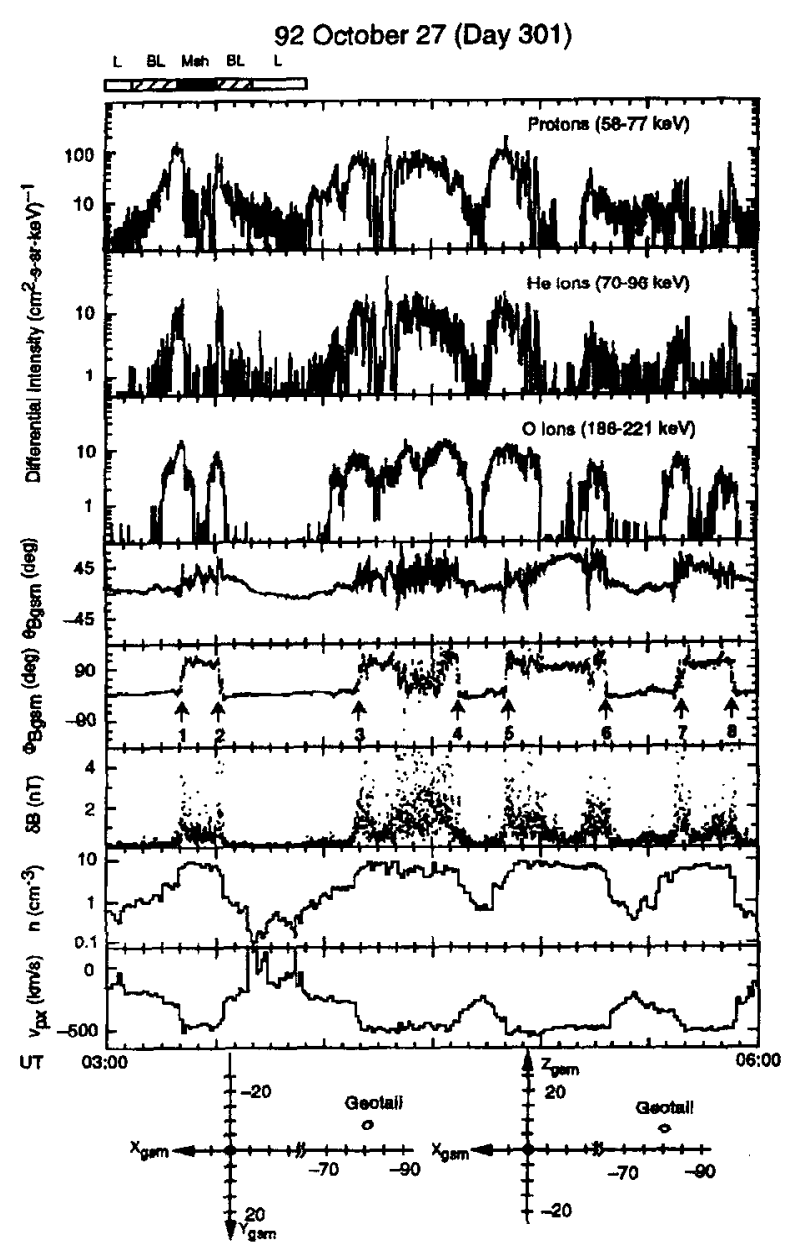

Fig. 1. Encounter of the distant tail boundary layer by Geotail on October 27, 1992. 
mum intensity of energetic particles occurred nearly simultaneous with an abrupt rotation of the magnetic field from a tail-like orientation to one of a draped field line on the magnetopause surface. At the second intensity peak, the magnetic field rotated back to a tail-like orientation. Within the two intensity peaks of energetic particle flux, the magnetic field became highly variable while the plasma density increased and its bulk flow became tailward at a high speed of $\sim 500 \mathrm{~km} / \mathrm{s}$. This time sequence of measurements is consistent with Geotail exiting the tail temporarily. During its exit and entry of the tail, Geotail encountered a boundary layer with its highest energetic particle flux at the magnetopause. In the boundary layer and magnetosheath, energetic ions exhibit a tailward streaming anisotropy in association with tailward plasma bulk flow. Plasma parameters were determined by the Comprehensive Plasma Instrument $/ 3 /$. The region identification is illustrated by the legend at the top of Fig. 1. The same interpretation applies well to all the enhancements of energetic particle flux in this period.

To gain further insight on the source of the energetic particles seen at the magnetopause boundary layer, we examined the composition and charge state information provided by STICS. Fig. 2 shows the mass and charge state of the measured ions during the 3-hour interval of Fig. 1. The presence of $\mathrm{H}^{+}, \mathrm{He}^{++}, \mathrm{He}^{+}, \mathrm{N}^{+}$, and $\mathrm{O}^{+}$ions is evident, indicating that both the ionosphere and the solar wind are important sources for the observed population of energetic particles in the magnetopause boundary layer.

Plasma data indicate that the lobe region was characterized by relatively slow $(<300 \mathrm{~km} / \mathrm{s})$ tailward moving plasma at energies of a few hundred $\mathrm{eV}$, suggesting that the tail lobe was populated by plasma mantle where energetic particle flux was near the background level. The plasma mantle was convecting towards the neutral sheet at $\sim 55 \mathrm{~km} / \mathrm{s}$. An important feature is that energetic particles were seen only at the magnetopause boundary and did not penetrate deep into the tail at this time. This feature allows Williams et al. $/ 2 /$ to put an upper limit on the distance from Geotail where encrgetic ions were accelerated or made contact with the magnetopause. The result shows that energetic ions of both solar wind and ionospheric origin started not

1992 October $27(03: 00-06: 00$ UT)

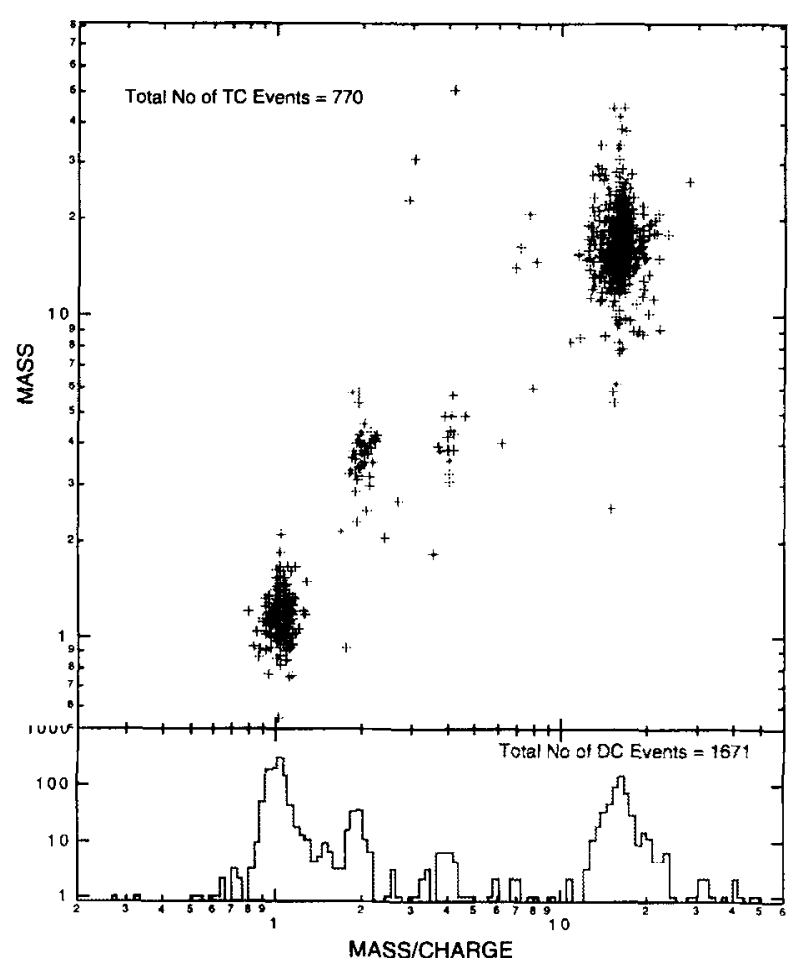

Fig. 2. Energetic ion species determined by EPIC. 
more than $\sim 40 R_{\mathrm{E}}$ earthward of Geotail, indicating that these particles were accelerated or made contact with the magnetopause many tens of earth radii behind the Earth.

We note that Geotail was at a distance of $\sim 11 R_{\mathrm{E}}$ from the GSM $x$-axis, which is rather unusual for encountering the magnetopause when its downstream distance was only $\sim 80 R_{\mathrm{E}}$. Possible causes for this are nonradial solar wind flow, decreased tail cross-section $/ 4 /$, propagation of a symmetric surface wave at the magnetopause $15 /$, and some convoluted tail topology $/ 6 /$. Fig. 3 examines the possibility of nonradial solar wind flow where the Geotail location and the aberrated tail axis locations based on solar wind flow direction monitored by IMP-8 are plotted for magnetopause crossings encountered by Geotail. Solar wind flow direction averaged over 30 min prior to each magnetopause crossing was used with the propagation delay time of $\sim 20 \mathrm{~min}$ between IMP-8 and Geotail taken into account. The errors associated with each aberrated tail axis location reflect the variability of the solar wind flow direction within the averaging interval. No aberrated tail axis is calculated for the magnetopause crossing \#3 because of a data gap in the corresponding solar wind flow direction. Arcs at various radial distances from the Geotail location are drawn to provide guidance in judging the dimension of the tail radius. For example, if the tail radius were $20 R_{\mathrm{E}}$ at a magnetopause crossing time, then the aberrated tail axis should lie on the arc at $20 R_{\mathrm{E}}$ from the Geotail location. Since the nominal tail radius at this downstream distance is $-22 R_{\mathrm{E}} / 4 /$, this figure indicates a decrease of the tail radius from nominal value for crossings $\# 2$, \#5, \#7, and \#8.

There is also an indication of a surface wave propagating down the nightside magnetopause during this interval. Fig. 4 shows the magnetopause surface normal projected in the $Y Z$-, $X Z$-, and $X Y$-planes. The normal was determined from magnetic field measurements by the minimum variance technique. The dashed line indicates the normal from the previous magnetopause crossing. This figure shows the normal rocking back and forth between successive crossings. Furthermore, the sense of the tilt in the normal between tail entry and exit is consistent with a surface wave at the magnetopause propagating tailward and towards the equatorial plane. The wave period inferred from times between successive entry or exit is long, $\sim 30-70 \mathrm{~min}$.

There is indication that energetic particles in the magnetopause boundary layer penetrate inside the tail occasionally. Fig. 5a-c shows enhancements in the energetic particle flux at the magnetopause crossings of Geotail similar to the ones encountered earlier on that day as given in Fig. 1. Lui et al. /7/ examined closely the energetic particle fluxes preceding the first magnetopause encounter at $\sim 1126$ UT and noted the presence of a brief flux reduction for protons and helium ions at $\sim 1118 \mathrm{UT}$ but not for oxygen ions. There are three

Aberrated Tail Axis at Magnetopause Crossings

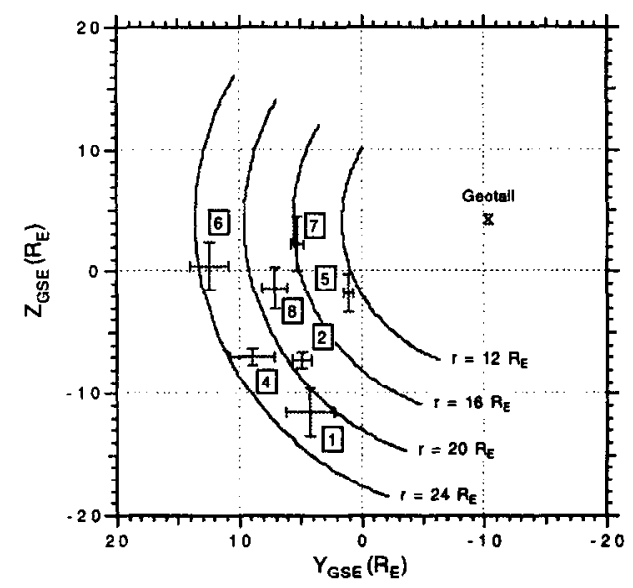

Fig. 3. Tail dimensions at Geotail magnetopause crossings on October 27, 1992. 


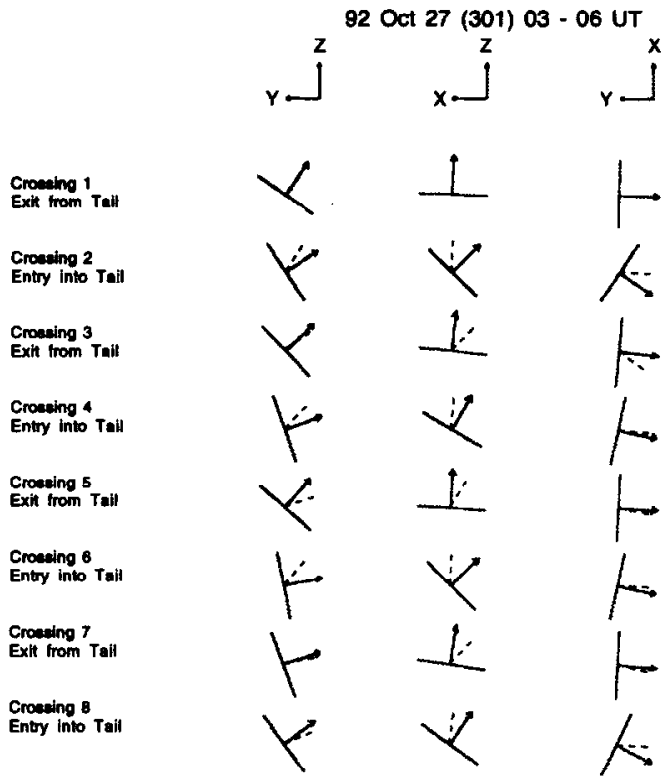

Fig. 4. Magnetopause boundary normal orientation during Geotail magnetopause crossings.

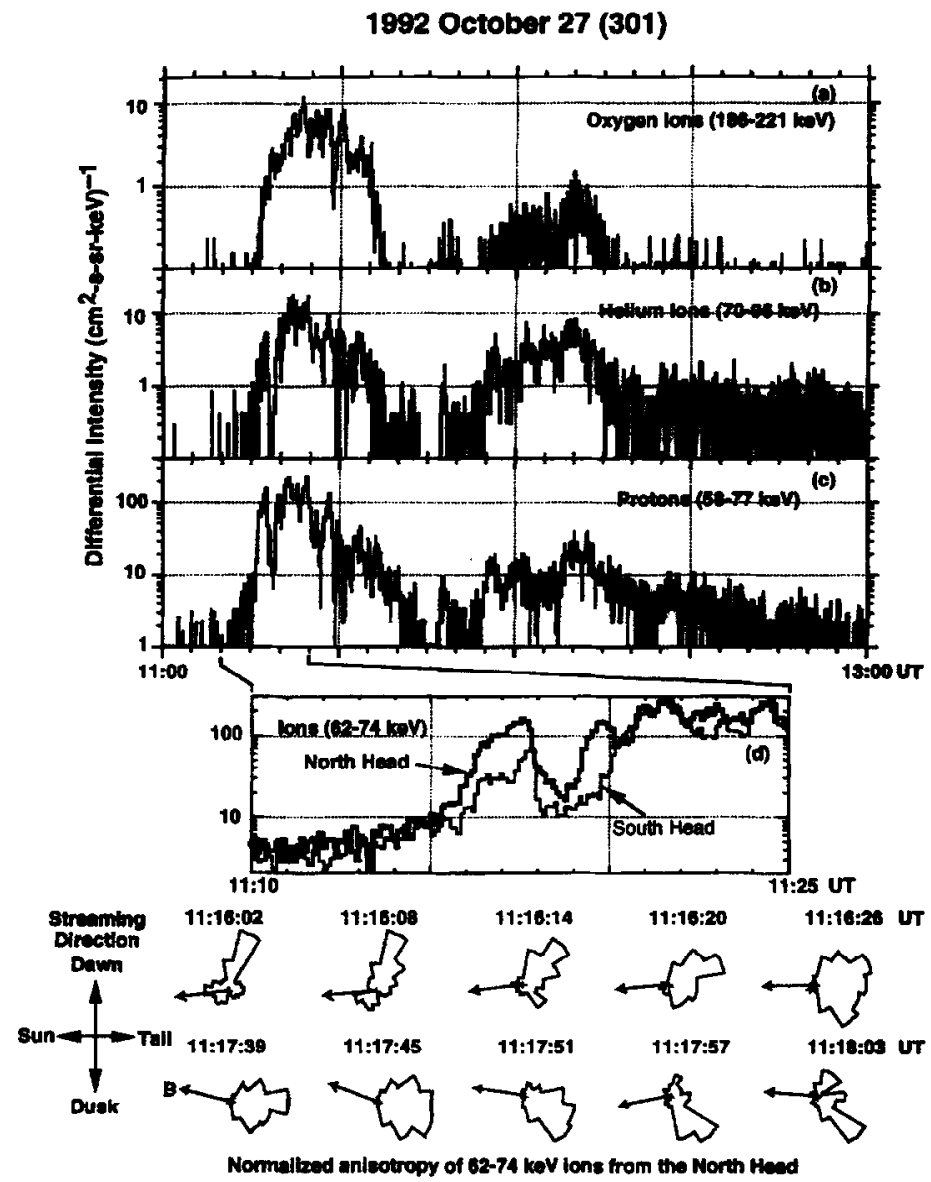

Fig. 5. A filament of energetic ions detached from the magnetopause boundary layer. 
possible causes of this flux reduction. It may indicate a temporal flux variation of the energetic particle boundary layer, or the boundary layer receding momentarily from the spacecraft in its approach, or a spatial gap between the magnetopause boundary layer and a region of energetic particles detached from the boundary layer. Resolving these possibilities is tantamount to differentiating temporal variation from spatial variation with observations from a single spacecraft. Fortunately, this seems realizable in this case.

Fig. 6 shows the expected response for the two viewing polar angles from these three possibilities. If it were a temporal variation, fluxes at both heads would vary synchronously. If it were due to a momentary retreat of the boundary layer, the south head flux would be nested within the north head flux. If it were a detached boundary layer passing through the spacecraft, the south head flux would resemble a time-shifted north head flux. The 62-74 keV energetic ion flux at two polar angles measured by ICS are shown separately in Fig. 5d, with the north viewing indicated by a thick line and south viewing by a thin line. It is evident that flux variation from the north viewing led that from the south viewing at both the enhancement and the reduction. As indicated in Fig. 6, this favors the interpretation of a layer of energetic particles moving from north to south across the spacecraft. This deduction is further supported by another independent assessment using the ion sounding technique for energetic particles /8/. At the bottom of Fig. 5 are two rows (for two different time intervals) of anisotropy plots displaying the azimuthal variation of ion flux at $62-74 \mathrm{keV}$ measured by the north head at $\sim 6$-s resolution. These anisotropy plots show the fluxes, normalized to the maximum flux for each sampling interval, in the particle streaming direction as indicated by the legend at the left side. The magnetic field direction projected on the spin plane of the spacecraft is given by the arrow in each plot. Since the magnetic field was pointing roughly sunward, particles having a dawnward (duskward) streaming component had gyrocenters north (south) of the spacecraft. With this understanding, the first plot at 11:16:02 UT indicates that the maximum flux came from particles having gyrocenters nearly one gyroradius north of the spacecraft. The particles then approached the spacecraft with time until the spacecraft was embedded in this layer at 11:16:26 UT. The second row shows the spacecraft's exit from this layer. The continuation of the clockwise rotation of the sector with the maximum flux indicates that the layer continued to move southward. This time sequence of anisotropy variation reinforces the interpretation given earlier based on the comparison of fluxes from the north and south heads that a filament of tailward streaming energetic particles, detached from the magnetopause boundary layer, moved from north to south over the spacecraft towards the neutral sheet.

A number of features associated with the detached filament can be inferred. The time interval over which the filament was sensed to move across the spacecraft can be used to estimate its north-south velocity. Using the information provided by the anisotropy plots, we find the average north-south speed to be $\sim 82 \pm 21 \mathrm{~km} / \mathrm{s}$. This value agrees well with the north-south plasma flow determined by the plasma instrument which showed the average plasma flow in the corresponding interval to be $\sim 80 \pm 15 \mathrm{~km} / \mathrm{s}$. If the north-south motion arises

\section{Expected Responses of North and South Heads}

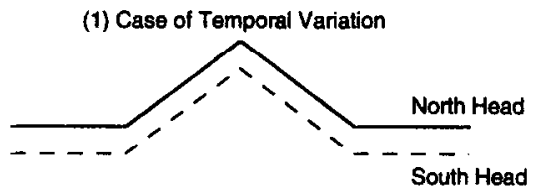

(2) Case of Momentary Retreat in Boundary Layer's Approach

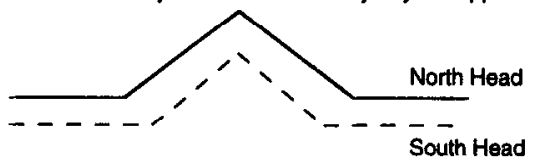

(3) Case of A Delached Boundary Layer Filament Passing Through the S/C

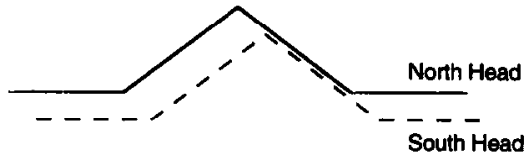

Fig. 6. Expected responses for north and south heads for three different possibilities. 
from a convective motion, then the average convection electric field can be deduced to be $\sim 1.0 \pm 0.3 \mathrm{mV} / \mathrm{m}$. During this interval, there was a transient impulsive enhancement of the duskward electric field component to $\sim 3 \mathrm{mV} / \mathrm{m}$. Averaged over the interval of the filament $(\sim 1116-1118 \mathrm{UT})$, the $y$-component electric field value was $0.71 \pm 0.18 \mathrm{mV} / \mathrm{m}$, agreeing reasonably well with the value inferred from the motion of the filament. We also deduce the north-south thickness of the filament to be $\sim 1.0 \pm 0.3 R_{\mathrm{E}}$. At later times, the gradient anisotropy associated with the filament was detected simultaneously with the gradient anisotropy associated with the energetic boundary layer, which offers an estimate of $\sim 0.8 \pm 0.1 R_{\mathrm{E}}$ for the separation distance between the northern edge of the filament and the southern edge of the boundary layer. This separation is smaller than the gyroradius of the $186-221 \mathrm{keV} \mathrm{O}^{+}$ions and is consistent with the observation that the flux of oxygen ions did not show a decrease at $-11: 18$ UT. The magnetic field direction changed from having a small duskward component before entering the filament to having a small dawnward component. This change in the field azimuth is consistent with an earthward field-aligned current system within the filament. The change in the dawn-dusk magnetic field component gives an estimate of $\sim 4.5 \pm 1.2 \mathrm{~mA} / \mathrm{m}$ as the north-south integrated current density within the filament.

For the two-hour period shown in Fig. 5a-c, Geotail entered the magnetosheath twice. The detached filament was detected prior to the first magnetosheath entry while no detached filament was revealed for the first exit of the magnetosheath nor for the second entry and exit. The lack of repeatable observation of the detached filament suggests it to be a transient feature occasionally detectable near the distant magnetopause and its occurrence is probably related to the process governing the entry of energetic particles into the magnetosphere through the high-latitude magnetopause.

\section{MOLECULAR IONS IN THE DISTANT TAIL}

While ionospheric ions like $\mathrm{O}^{+}$and $\mathrm{He}^{+}$can contribute significantly to the energetic particle population, molecular ions are often below background flux levels (Fig. 2). However, during high geomagnetic activity conditions, their presence can be detected in the distant tail, as found by Christon et al. /9/. Such is the case during 0930-1330 UT on 1993 October 9 when $\mathrm{K}_{\mathrm{p}}$ was $5^{+}$and $7^{-}$. Geotail was located at $(-146,14,3) R_{\mathrm{E}}$ in GSM coordinates when it entered the plasma sheet at 0937 UT. At 0957 UT, the ion flux level increased abruptly while plasma flow speed increased gradually and the flow direction turned tailward. After the start of strong tailward plasma flow at 1002:30 UT, molecular ions were detected rather continuously for $\sim 25$ min. Ion composition was primarily $\mathrm{H}^{+}, \mathrm{He}^{++}, \mathrm{N}^{+}$, and $\mathrm{O}^{+}$throughout, with $\mathrm{H}^{+}$dominating. It was an extremely dynamic plasma sheet interval in which complex flow patterns were observed at high temporal resolution. Fig. 7 shows the histograms of the mass-per-charge frequency distribution at energy/charge values of 136 and $87 \mathrm{keV} / \mathrm{e}$ for all pulse height analyzed events measured within $\sim 45^{\circ}$ azimuth of the $x$-axis by STICS for the two time intervals 0958-1026 UT and 1252-1330 UT. Molecular ions, labeled as MI, appear at the far right of the plot at M/Q values of $\sim 28-32 \mathrm{amu} / \mathrm{e}$. The bottom panels show the phase space densities in the average plasma rest frame when the bulk flow speed was $\sim 350 \mathrm{~km} / \mathrm{s}$. This result indicates that $\mathrm{H}^{+}$was the dominant energetic ion in the flow, $\mathrm{He}^{++}$and $\mathrm{O}^{+}$abundance were comparable, and the estimated ratios $\mathrm{N}^{+} / \mathrm{O}^{+}$and $\left(\mathrm{O}_{2}^{+}+\mathrm{NO}^{+}+\mathrm{N}_{2}^{+}\right) / \mathrm{O}^{+}$are $\sim 25 \%$ and $\sim 2 \%$, respectively.

\section{TAILWARD ENERGETIC ION STREAMING DURING GEOMAGNETIC ACTIVITY}

During a moderately active period between 2300 UT on January 20, 1993 and 1200 UT on January 21, 1993 Geotail was located from $(-94,3,-5) R_{\mathrm{E}}$ to $(-95,0,-6) R_{\mathrm{E}}$ in GSM coordinates and there were several substorm intensifications recorded on the ground. Fig. 8, taken from Jacquey et al. $/ 10 /$, shows the observations from Geotail. The bottom panel plots the ratio of the sunward over the tailward ion fluxes for 74-89 $\mathrm{keV}$ ions. A ratio $>1$ indicates earthward streaming while $<1$ indicates tailward streaming. Onsets/ intensifications of the near-Earth geomagnetic activity are represented by the vertical dotted lines. High fluxes of energetic particles were seen at large and small values of IBXI, suggesting that the flux changes were due largely to temporal effects rather than to spatial variations. At 2344 UT on January 20, 1993 a sharp increase in energetic ion flux was observed, reaching the peak flux at 0009 UT the next day. This enhancement was closely related to the times 2348 UT and 0009 UT of the onset/intensification on the ground. 

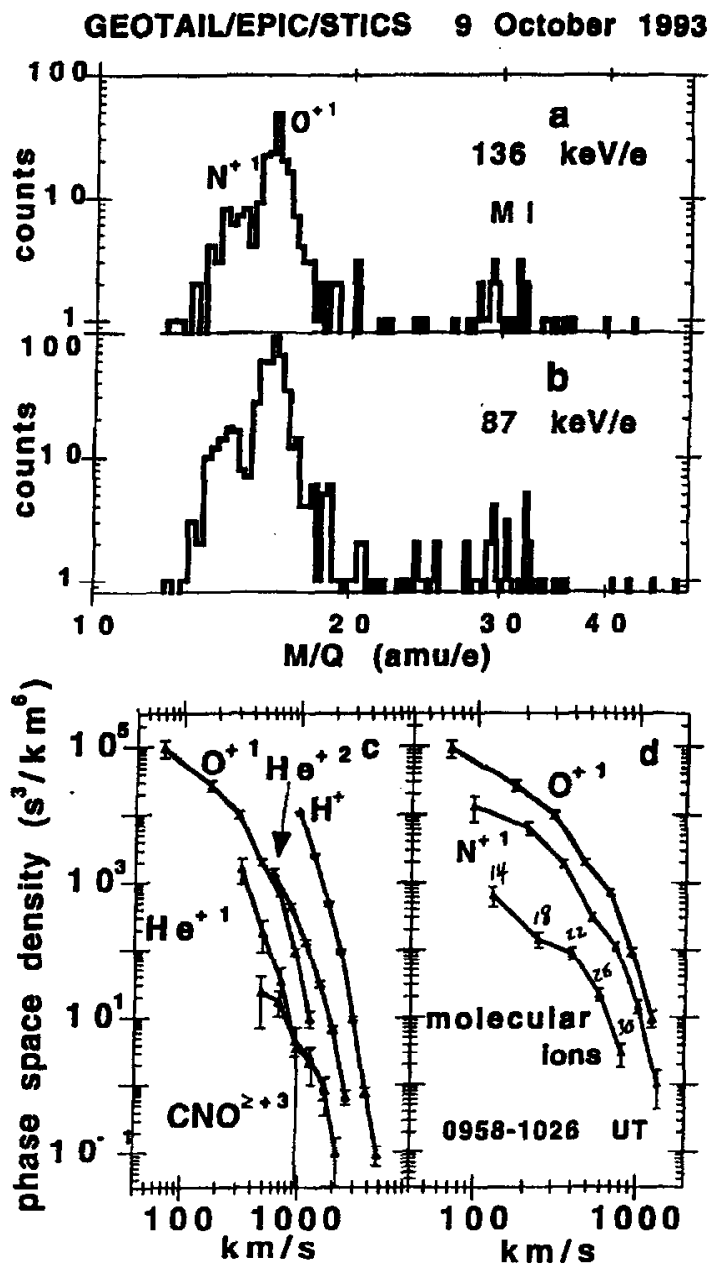

Fig. 7. Observation of molecular ions by EPIC.

Another flux increase occurred at 0303 UT, which corresponded to the gradual development of negative bays at the ground between 0300 and 0315 UT. Later on, ground activity indicated abrupt auroral electrojet intensifications at 0403 and 0415 UT. The time for the second intensification corresponded well with a recovery of energetic particle flux in the tail. The ground activity at 0849 UT did not seem to match with an increase in the energetic ion flux. On the other hand, the ground activity at 1003 UT corresponded within $\sim 2$ min to the onset of tailward streaming of energetic ions at Geotail. In general, there seems to be a correlation between the amount of energetic ion flux increase and ground substorm intensity. Since it is difficult to determine substorm onset accurately from ground magnetograms, the above correlation needs further examination in the future.

\section{SUMMARY}

Some initial results from Geotail based primarily on EPIC measurements are reported here. The distant tail is found to be a complex region with in situ particle acceleration and particle entry from the magnetosheath to the tail. Energetic ions of both solar wind and ionosphere origin populate the distant tail. Molecular ions are detectable at high geomagnetic activity. There is a close time correlation between onsets of geomagnetic activity at the Earth and the increase in the energetic ion flux in the distant tail, indicating an intimate coupling between the near-Earth plasma and the distant tail plasma. Future investigations will provide more insights on the distant tail dynamics and its coupling to the near-Earth region. 


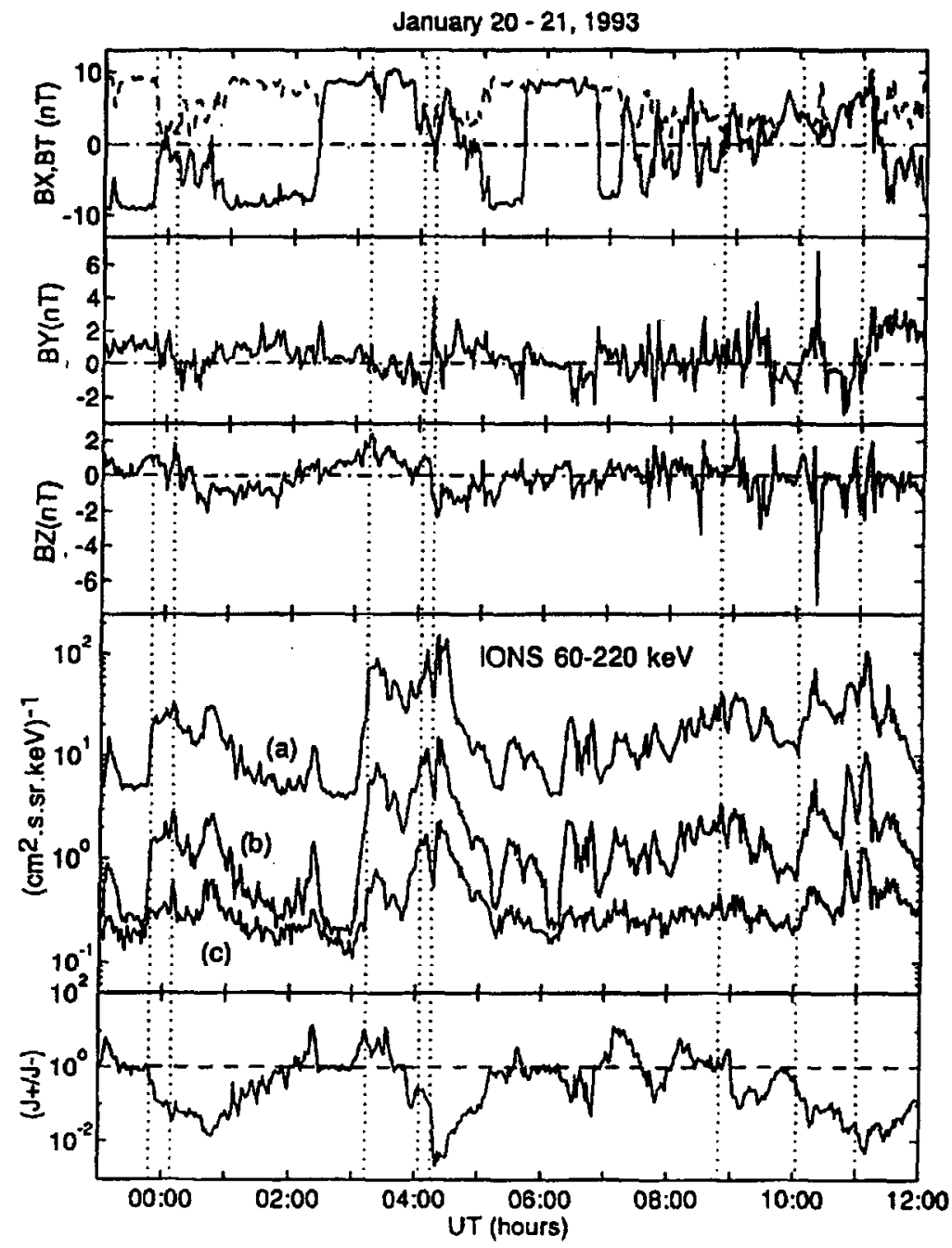

Fig. 8. Geotail observations from 2300 UT on January 20, 1993 to 1200 UT on January 21, 1993.

\section{ACKNOWLEDGMENTS}

This work was supported by the Space Physics Division of the National Aeronautics and Space Administration to The Johns Hopkins University Applied Physics Laboratory under the Department of Navy Task IAF; Contract N00039-91-C-001. The research at the University of Iowa was supported in part by NASA under contract NAS5-26858.

\section{REFERENCES}

1. D. J. Williams, R. W. McEntire, C. Schlemm II, A. T. Y. Lui, G. Gloeckler, S. P. Christon, and F. Gliem, Geotail energetic particles and ion composition instrument, J. Geomag. Geoelectr., 46, 39, 1994.

2. D. J. Williams, A. T. Y. Lui, R. W. McEntire, V. Angelopoulos, C. Jacquey, S. P. Christon, L. A. Frank, K. L. Ackerson, W. R. Paterson, S. Kokubun, T. Yamamoto, and D. H. Fairfield, Magnetopause encounters in the magnetotail at distances of $\sim 80 R_{\mathrm{E}}$, Geophys. Res. Lett., in press, 1994. 
3. L. A. Frank, K. L. Ackerson, W. R. Paterson, J. A. Lee, M. R. English, and G. L. Pickett, The Comprehensive Plasma Instrumentation (CPI) for the GEOTAIL spacecraft, J. Geomag. Geoelectr., 46, 23, 1994.

4. D. H. Fairfield, Solar wind control of the distant magnetotail: ISEE 3, GSFC LEP Preprint, February 1993.

5. G. Siscoe, L. A. Frank, K. L. Ackerson, W. R. Paterson, and M. Ashour-Abdalla, Interpretation of longperiod, velocity dispersive plasma events at $100 R_{\mathrm{E}}$ and beyond in terms of comparison with an expansion fan model, EOS Trans. Am. Geophys. Union, 74, 43, 528, 1993.

6. M. Ashour-Abdalla, L. A. Frank, W. R. Paterson, K. L. Ackerson, F. V. Coroniti, L. M. Zelenyi, S. Kokubun, D. H. Fairfield, and A. J. Lazarus, On cold ion beams in the distant tail, EOS Trans. Am. Geophys. Union, 74 , 43, 528, 1993.

7. A. T. Y. Lui, D. J. Williams, R. W. McEntire, S. P. Christon, C. Jacquey, V. Angelopoulos, T. Yamamoto, S. Kokubun, L. A. Frank, K. L. Ackerson, and K. Tsuruda, A filament of energetic particles near the highlatitude dawn magnetopause, Geophys. Res. Lett., in press, 1994.

8. D. J. Williams, T. A. Fritz, B. Wilken, and E. Keppler, An energetic particle perspective of the magnetopause, J. Geophys. Res., 84, 6385, 1979.

9. S. P. Christon, G. Gloeckler, D. J. Williams, T. Mukai, R. W. McEntire, C. Jacquey, V. Angelopoulos, A. T. Y. Lui, S. Kokubun, T. Yamamoto, and D. H. Fairfield, Energetic atomic and molecular ions of ionospheric origin observed in distant magnetotail flow-reversal events, Geophys. Res. Lett., in press, 1994.

10. C. Jacquey, D. J. Williams, R. W. McEntire, A. T. Y. Lui, V. Angelopoulos, S. P. Christon, D. H. Fairfield, S. Kokubun, T. Yamamoto, G. D. Reeves, and R. D. Belian, Tailward energetic ion streams observed at $\sim 100$ $R_{\mathrm{E}}$ by Geotail-EPIC associated with geomagnetic activity intensifications, Geophys. Res. Lett., in press, 1994. 